\title{
Examining publication bias - A simulation-based evaluation of statistical tests on publication bias
}

\author{
Andreas Schneck ${ }^{\text {Corresp. } 1}$ \\ ${ }^{1}$ Department of Sociology, Ludwig-Maximilians-Universität München, Munich, Germany \\ Corresponding Author: Andreas Schneck \\ Email address: andreas.schneck@Imu.de
}

\section{Background}

Publication bias is a form of scientific misconduct. It threatens the validity of research results and the credibility of science. Although several tests on publication bias exist, no in-depth evaluations are available that examine which test performs best for different research settings.

\section{Methods}

In the study at hand four tests on publication bias, Egger's test (FAT), p-uniform, the test of excess significance (TES), as well as the caliper test, were evaluated in a Monte Carlo simulation. Two different types of publication bias and its degree $(0 \%, 50 \%, 100 \%)$, were simulated. The type of publication bias was defined either as file-drawer, meaning the repeated analysis of new datasets, or $p$-hacking, meaning the inclusion of covariates in order to obtain a significant result. In addition, the underlying effect $(\beta=0$, $0.5,1,1.5)$, effect heterogeneity, the number of observations in the simulated primary studies $(N=100$, $500)$, and the number of observations for the publication bias tests ( $K=100,1000)$, were varied.

\section{Results}

All tests evaluated were able to identify publication bias both in the file-drawer and p-hacking condition. The false positive rates were, with the exception of the $15 \%$ - and $20 \%$-caliper test, unbiased. The FAT had the largest statistical power in the file-drawer conditions, whereas under $p$-hacking the TES was, except under effect heterogeneity, slightly better. The CTs were, however, inferior to the other tests under effect homogeneity and had a decent statistical power only in conditions with 1000 primary studies.

\section{Discussion}

The FAT is recommended as a test for publication bias in standard meta-analyses with no or only small effect heterogeneity. If two-sided publication bias is suspected as well as under $p$-hacking the TES is the first alternative to the FAT. The $5 \%$-caliper test is recommended under conditions of effect heterogeneity and a large number of primary studies, which may be found if publication bias is examined in a discipline-wide setting when primary studies cover different research problems. 
1 Examining publication bias - A simulation-based evaluation 2 of statistical tests on publication bias

3 Andreas Schneck ${ }^{1}$

$4{ }^{1}$ Department of Sociology, Ludwig-Maximilians-Universität München, Munich, Germany

5 Corresponding Author:

6 Andreas Schneck ${ }^{1}$

7 Konradstraße 6, Munich, 80801, Germany

8 Email address: andreas.schneck@1mu.de 


\section{Abstract}

\section{Background}

11 Publication bias is a form of scientific misconduct. It threatens the validity of research results and

12 the credibility of science. Although several tests on publication bias exist, no in-depth evaluations

13 are available that examine which test performs best for different research settings.

\section{Methods}

15 In the study at hand four tests on publication bias, Egger's test (FAT), p-uniform, the test of 16 excess significance (TES), as well as the caliper test, were evaluated in a Monte Carlo simulation.

17 Two different types of publication bias and its degree $(0 \%, 50 \%, 100 \%)$, were simulated. The 18 type of publication bias was defined either as file-drawer, meaning the repeated analysis of new 19 datasets, or p-hacking, meaning the inclusion of covariates in order to obtain a significant result. 20 In addition, the underlying effect $(\beta=0,0.5,1,1.5)$, effect heterogeneity, the number of 21 observations in the simulated primary studies $(N=100,500)$, and the number of observations for 22 the publication bias tests $(K=100,1000)$, were varied.

\section{Results}

24 All tests evaluated were able to identify publication bias both in the file-drawer and p-hacking 25 condition. The false positive rates were, with the exception of the $15 \%$ - and $20 \%$-caliper test, 26 unbiased. The FAT had the largest statistical power in the file-drawer conditions, whereas under 27 p-hacking the TES was, except under effect heterogeneity, slightly better. The CTs were, 28 however, inferior to the other tests under effect homogeneity and had a decent statistical power 29 only in conditions with 1000 primary studies.

\section{Discussion}


31 The FAT is recommended as a test for publication bias in standard meta-analyses with no or only

32 small effect heterogeneity. If two-sided publication bias is suspected as well as under p-hacking

33 the TES is the first alternative to the FAT. The 5\%-caliper test is recommended under conditions

34 of effect heterogeneity and a large number of primary studies, which may be found if publication

35 bias is examined in a discipline-wide setting when primary studies cover different research 36 problems. 
37

\section{Introduction}

All scientific disciplines try to uncover truth by systematically examining their surrounding environment (Descartes 2006: 17). Natural scientists try to observe regularities in nature, whereas social scientists try to uncover patterns in the social behaviour of humans. The success, as well as the reputation, of science rests on the accuracy and unbiasedness of scientific results. Publication bias, the publication of only positive results confirming the researcher's hypothesis (cf. Dickersin \& Min 1993: 135), threatens this validity. Under publication bias only results showing either statistical significance and/or the desired direction of the effects are published. The published literature in this case is merely a selective (and too optimistic) part of all existing scientific knowledge. Furthermore, science is in the case of publication bias also inefficient as studies that add substantial knowledge to the literature, but contain null-findings remain unpublished.

The study at hand examines the performance of four methods to identify publication bias: Egger's Test/FAT (Egger et al. 1997; Stanley \& Doucouliagos 2014), p-uniform (PU; van Aert et al. 2016; van Assen et al. 2015), the test for excess significance (TES; Ioannidis \& Trikalinos 2007) and the caliper test (CT; Gerber \& Malhotra 2008a; Gerber \& Malhotra 2008b). In order to compare the performance of these tests, the false positive rate ( $\alpha$-error, type I error) and the statistical power (true positive rate) were examined in a Monte Carlo simulation study. This makes it possible to assess the performance of the four tests under different conditions of publication bias (file-drawer vs. p-hacking), as well as study settings (underlying true effect, effect heterogeneity, number of observations in primary studies and in meta-analyses).

\section{The issue of publication bias}

The false positive rate of a test (commonly called $p$-value) is the probability of the estimator rejecting $\mathrm{H}_{0}$ despite this being true. The $p$-value is therefore the probability that the observed estimate is at least as extreme given there is no effect as assumed by $\mathrm{H}_{0}$ (Wasserstein \& Lazar 
61 2016). The larger the $p$-value the higher the risk of assuming an effect if none exists in the data.

$62 p$-values below a certain threshold are called statistically significant, whereas values above the

63 threshold are labelled as non-significant. In the empirical sciences the 5\%-significance threshold

64 is mostly used (Cohen 1994; Labovitz 1972; Nuzzo 2014). The difference between 0.049 and

650.051 in the error probability is, however, marginal. Nevertheless, from the standpoint of the 5\%-

66 significance threshold the first would be a significant effect, whereas the latter would be a non-

67 significant effect. In both of these two cases on average around 1 in 20 null-hypotheses of no

68 difference would be rejected, albeit true. If empirical researchers select their data/models until

69 they find, just by chance, significant evidence that seems worth publishing, publication bias is on

70 the rise, leading to inflated or even artificial effects.

71 Rosenthal (1979) constructs a worst case scenario in which only the $5 \%$ of false positive studies

72 that are "significant" solely by pure chance are published. In this case, misinterpreted results

73 shape the scientific discourse and finally result in (medical or political) interventions. Although

74 Rosenthal's example is extreme, a multitude of evidence for publication bias exists in various

75 disciplines and research fields (e.g. Doucouliagos \& Stanley 2009; Jefferson et al. 2012). Godlee

76 (2012) therefore warns that scientific misconduct, under which publication bias is subsumed

77 (Chalmers 1990), may also physically harms patients.

78 In addition to the societal consequences, publication bias also has severe implications for the

79 evolution of knowledge. Under publication bias no rejection of theories (Popper 1968: 215), on

80 which all scientific progress relies, occur which leads to a state of "undead theory" (Ferguson \&

81 Heene 2012: 559) where all existing theories are confirmed irrespective of their truth.

82 Motivation to commit publication bias

83 Because statistically significant results stress the originality of research findings (Merton 1957),

84 Both, authors and scientific journals (cf. Coursol \& Wagner 1986; Epstein 1990, 2004; Mahoney 
85 1977) have large incentives to maximise their significant results to survive in a publish or perish

86 research environment. Authors especially want to increase their publication chances, notably in

87 top-tier journals where low acceptance rates of $5 \%-10 \%$ are quite common (for the top

88 interdisciplinary journals Nature 2017; Science 2017; cf. for the political sciences Yoder \&

89 Bramlett 2011: 266). Two distinct strategies to achieve significant results by means of publication

90 bias practices can be pointed out. Firstly, non-significant findings can be suppressed (cf. the

91 classical file-drawer effect described by Rosenthal 1979) and significant results are then searched

92 for in another dataset. Secondly, small bits in the data analysis can be changed (e.g. adding

93 covariates, optional stopping, exclusion of outliers, etc.) until a significant result is obtained -

94 this method is known as p-hacking (cf. "fishing" Gelman 2013; or "researchers degree of

95 freedom" Simmons et al. 2011: 1359). Whereas the file-drawer strategy can be utilised by authors

96 as well as by editors and reviewers, p-hacking can only be committed by authors/researchers.

97 Nonetheless, p-hacking strategies can be recommended by actors other than authors (e.g. editors,

98 reviewers, etc.). Especially p-hacking is almost without any costs, as data analysis tools/packages

99 become increasingly easy to apply (Paldam 2013).

100 Evidence on the prevalence of publication bias

101 So far, there are two strategies for identifying publication bias: the first traces studies through the

102 publication process, the second asks authors, reviewers, or editors about their publication

103 practices via surveys. In the first strategy, most of the analyses trace conference papers or ethics

104 committee decisions if those results get published or remain in the file-drawer. Overall previous

105 findings note, that studies with significant results have a substantially higher chance to get

106 published (cf. Callaham et al. 1998; Coursol \& Wagner 1986; Dickersin 1990; Easterbrook et al.

107 1991). Ioannidis (1998) in addition finds that significant studies have, beside their higher

108 publication rate, also a substantially higher publication speed, meaning a shorter time between the 
109 completion of the study and the final publication. This results suggest, that publication bias is a

110 beneficial strategy in order to maximize academic merits.

111 The second approach asks directly about the publication practices of the involved actors. In a

112 survey of psychologists that used a sensitive question technique up to $50 \%$ of the respondents

113 claimed that they exercised publication bias (John et al. 2012: 525). Franco et al. (2014) also note

114 that most non-significant findings go to the file-drawer right after the analysis and are not even

115 written up. Also, other forms of misbehaviour, like optional stopping (stopping data collection

116 when significance is reached) or erroneous rounding of $p$-values to reach significant results, are

117 alarmingly widespread (prevalence rate arround 22.5\% John et al. 2012: 525). These results are in

118 line with the survey of Ulrich \& Miller (2017: 9), who report that researchers in the field of

119 psychology prefer significant results over non-significant results, and, furthermore, attribute more

120 value to results with smaller $p$-values. These estimates may even be conservative because it is

121 known from the survey literature that sensitive behaviours like scientific misconduct may be

122 underreported (Kreuter et al. 2008: 848). According to the presented research results file-drawer

123 and p-hacking behaviour is therefore quite widespread.

\section{Methods}

125 Publication bias tests in comparison

126 So far, the presented detection strategies ask either directly for publication preferences or

127 examine the publication fate of conference papers. Both approaches have the weakness that they

128 either rely on the potentially biased answers of the actors involved or require an immense effort

129 to follow the publication process, while publication bias may have happened before the paper is

130 submitted to a conference. Statistical tests on publication bias circumvent this problem by relying

131 only on the published literature. In the paper at hand the regression-based FAT (Egger et al. 1997;

132 Stanley \& Doucouliagos 2014), PU (van Aert et al. 2016; van Assen et al. 2015), an extended 
133 version of p-curve (Simonsohn et al. 2014a, b; Simonsohn et al. 2015), the TES (Ioannidis \&

134 Trikalinos 2007), and the CT (Gerber \& Malhotra 2008a, b) were evaluated (see online appendix

135 for an in-depth discussion of the tests). ${ }^{1}$

136 In order to compare the different publication bias tests, four different criteria have to be 137 established: the assumptions of the test, the measurement level, the sample used, the test method, 138 and its according limitations (see Table 1).

139 The FAT tests basically the relationship between study's precision and its effect size with all 140 available effect sizes from primary studies. If larger effects are observed for studies with low 141 precision (and low $N$ ) publication bias is suspected. Nonetheless if alternative reasons may lead 142 to this result: small studies examine specific high risk populations in which treatments may be 143 more effective (Sterne et al. 2011), this effect heterogeneity may lead to the diagnosis of 144 publication bias where none exists (Schwarzer et al. 2002). ${ }^{2}$ The FAT has furthermore the 145 disadvantage that only one-sided publication bias either in favour of a positive or negative 146 significant effect can be tested. Alinaghi \& Reed (2016: 10) show that if significant results of 147 either sign are searched for, the FAT suffers from massively inflated false positive rates. In the 148 Monte Carlo simulation at hand only the FAT is used because of its better statistical power as 149 shown in prior simulations compared to the similar rank correlation test of Begg \& Mazumdar $150 \quad(1994)^{3}$ and the trim and fill technique (Duval \& Tweedie 2000) ${ }^{4}$.

11 Because for Fail-save-N (Rosenthal 1979) only rules of thumbs, instead of a formal statistical test, exist it was not 2 included in the simulation at hand. Although it is still widely applied (Banks et al. 2012: 183; Ferguson \& Brannick 3 2012: 4), this benchmark is not recommended in the Cochrane Handbook, a guideline for conducting meta-analyses 4 (Higgins \& Green 2008: 321f.).

52 For a similar result see Terrin et al. (2003) for the related Trim and Fill technique (Duval \& Tweedie 2000).

63 For simulations see: Hayashino et al. (2005); Kicinski (2014); Macaskill et al. (2001); Sterne et al. (2000).

74 For simulations see: Bürkner \& Doebler (2014); Kicinski (2014); Moreno et al. (2009); Renkewitz \& Keiner 8 (2016). 
151 PU has the assumption that every left skewness in the distribution of $p$-values smaller than the

152 significance threshold (e.g. $p<0.05)$ and conditioned on the underlying observed mean effect 153 ( $p p$-value) distribution is caused by publication bias. This assumption is, however, grounded

154 mainly on the fixed-effect estimate of the mean effect, which is very sensitive to effect 155 heterogeneity. PU furthermore limits its test-value only on significant estimates in the direction 156 where publication bias is suspected (van Aert et al. 2016: 727). Therefore PU is, as the FAT, only 157 able to identify one-sided publication bias.

158 The TES (Ioannidis \& Trikalinos 2007; also called ic-index see Schimmack 2012) in contrast 159 relies only on a dichotomous classifier, testing if the number of expected significant results and 160 the empirically observed number of significant effects differ. Because the TES relies, as PU on 161 the fixed-effect estimate of the mean effect of all included studies it is sensible to effect 162 heterogeneity. A large controversy in the literature is not about the TES itself, but on its 163 application. Francis (2012a, b, c, d, e, 2013) used the TES to identify singular articles in order to 164 test if they suffer from publication bias. This may invalidate the assumption of independence 165 (Morey 2013: 181) as well as inflate the false positive rate in a similar manner than in primary 166 research (cp. HARKing Kerr 1998) if the TES is used in such an exploratory manner (Simonsohn 167 2013: 175). Ioannidis however responds that if the TES is applied on prespecified research 168 questions with a large and independent number of effect sizes, the TES is even a conservative test 169 on publication bias (Ioannidis 2013: 185).

170 The CT uses the most limited sample of the included tests that includes only estimates slightly 171 over and under the chosen significance level in a distribution of $z$-values. In case of publication 172 bias the assumption of a continuous distribution that results in an approximately even distribution 173 in a narrow interval (caliper) is violated by an overrepresentation of just significant results. The 174 broader the interval is set the more it may deviate from the assumed even distribution caused by 
175 the true underlying effect. This restrictive sample has the downside that the exclusion of most

176 available values may drastically reduce the statistical power of the test.

177 In contrast to the FAT, the other tests are only able to test for publication bias on pre-specified

178 levels (e.g. 0.05). Because the TES and the CT focus only on dichotomous classifiers (significant

179 or not in the case of the TES, slightly over or under the threshold for the CT) also tests on two-

180 sided publication bias are possible.

181 In previous simulation studies with a low number of included studies as well as observations PU

182 was superior to the TES (van Assen et al. 2015: 303) and the FAT (Renkewitz \& Keiner 2016).

183 However no evaluations exists based on a larger number of primary studies. In particular, the

184 newer publication bias tests like PU, the TES, and the CT, are in need of an evaluation under 185 different conditions. For the CT also no studies exist regarding the best caliper width to use.

186 Despite the existence of some simulation studies on publication bias tests, so far no direct 187 comparison exists that evaluates the performance of all four publication bias tests, especially 188 under effect heterogeneity.

189 Simulation setup

190 In order to examine the performance of the four publication bias tests, a Monte Carlo simulation 191 approach is used. For the simulation two different processes have to be distinguished: firstly, the 192 data generation process (DGP), and, secondly, the meta-analytical estimation method (EM). The 193 DGP provides the ground for the hypothetical data used by the simulated actors, as well as the 194 results they report, whereas the EM applies the tests on publication bias reported in the previous 195 section. The central advantage of using Monte Carlo simulations is that controlling the DGP 196 allows to identify which simulated studies suffer from publication bias and which do not. Similar 197 to the case in experiments, different conditions can be defined to ensure a controlled setting. The 198 performance of the estimators can then be examined under the different conditions. 
199 Data setup of the primary studies and meta-analyses

200 The first step of the DGP defines different effect size conditions that underlie the analyses of the

201 simulated actors (see Table 2). As a first condition the underlying true effect was specified by a

202 linear relationship with $\beta=0,0.5,1.0,1.5$. Analogous to a linear regression model this means for $203 \beta=0.5$, that an increase of one unit of the independent variable $\mathrm{x}$ increases the dependent 204 variable $y$ by 0.5 . The specified linear relationship between the dependent variable $y$ and the 205 independent variable $x$ had a normally distributed regression error term of $\varepsilon=\mathrm{N}(0,10)$, while the 206 variation of the independent variable was defined as $\sigma_{x}=2$ (for a similar setup see Alinaghi \& 207 Reed 2016; Paldam 2015). The regression coefficients can also be transformed in the Pearson 208 correlation coefficient yielding approximately $r=0,0.1,0.2,0.3$. This results are equivalent to 209 low or medium effect sizes in terms of Cohen (1992) and cover about $75 \%$ of the empirical 210 observed effects in psychology (Bosco et al. 2015: 436). ${ }^{5}$ In addition to the homogenous 211 conditions with a common effect size, a heterogeneous condition was added that assumes no 212 fixed distribution of an underlying effect but a uniform mixture of all four effect sizes, as defined 213 above, plus an additional effect of $\beta=2.0(r=0.4)$ in order to ensure enough variation.

214 As the FAT is based on study precision, which is mainly driven by the number of observations $215(N)$ of the primary studies, $N$ was computed as a second condition by an absolute normal 216 distribution with a mean of $100($ small $N$ ) or 500 (large $N)$ and a standard deviation of 150 . In 217 order to ensure an adequate statistical analysis for the primary studies, $N$ s equal to or smaller than 21830 were excluded. This procedure resulted in a right skewed distribution with a mean $N$ of 219 roughly 500 for the large $N$, and 165 for the small $N$ condition. The small $N$ condition reflects the 220 observed number of observations in leading economics journals (mean: 152, own computations

221 from the publicly available dataset of Brodeur et al. 2016) as well as of typical trials included in

$9516.6 \%$ of the simulated studies were adequately powered with at least $80 \%$ power (Cohen 1988: 56 ). The setting 10 produced by the DGP also reflects the results of (Ioannidis et al. 2017: 245), who report that only 10\% of the studies 11 in Economics are adequately powered. 
222 Cochrane reviews (mean: 118, Mallett \& Clarke 2002: 822). Because both studies refer mainly to

223 an experimental literature, the large $N$ condition reflects the more common number of 224 observations especially in ex-post-facto designs (e.g. survey studies).

225 The heterogeneity of effects in each of the meta-analyses was measured by $I^{2}$, the share of 226 systematic variation in respect to the overall variation consisting of the systematic and random 227 variation (Higgins \& Thompson 2002). In case of the small $N 68.62 \%$ and for a large $N 86.86 \%$ 228 of the variation was systematic in the heterogeneous effect condition. In terms of Higgins \& 229 Thompson (2002: 1553), an $I^{2}$ larger than 50\% has to be modelled explicitly in meta-analyses and 230 cannot be ignored.

231 In addition to the number of observations in the primary studies $(N)$ the number of primary 232 studies that were included in the meta-analysis and form the basis of the publication bias tests $(K)$ 233 was varied in the third condition. A setting with 100 studies was used as a lower condition, 234 whereas 1000 studies were set as an upper condition. Although on average the number of trials in 235 a meta-analysis is usually much lower than 100 studies (median 28 studies in the meta-meta236 analysis by Elia et al. 2016: 5). 100 studies were chosen because in this setting every publication 237 bias test evaluated is at least partially applicable. In other research areas like Economics, where 238 meta-regression models are more widely used to model effect heterogeneity, also higher numbers 239 of included trials estimates are quite common (e.g. 1474 effect estimates in Doucouliagos \& 240 Stanley 2009).

241 Behavioural setup of publication bias

242 Building on this data setup stage of the DGP the behavioural setup adds publication bias to the 243 simulation in a fourth step (see Table 2). Publication bias was defined as the willingness to collect 244 new data or run additional analyses if statistical significance failed $(p \geq 0.05)$ or a negative effect 245 occurred. In the simulation only one-sided publication bias was modelled because both, the FAT 
246 and PU are not able to model two-sided publication bias that focuses only on significant results

247 irrespective of its sign. It is important to note, that only the intent to commit publication bias was

248 varied in the simulation setup. The actual publication bias depends on the data setup itself: how

249 large is the true effect size $(\beta)$ and the number of observations $(N)$ in the primary studies? Or, in 250 short: is there already a significant positive result which does not need a publication bias 251 treatment?

252 Five different publication bias conditions have to be distinguished. Firstly, the condition without 253 publication bias: in this ideal case all estimates $(\beta x)$ are estimated by a bivariate ordinary least 254 squares (OLS) model and afterwards published. Publishing in terms of the simulation model 255 means that all estimates enter the final meta-analysis. Therefore, in the condition without 256 publication bias either 100 or 1000 regression results were estimated and enter the meta-analysis.

257 In the second and third conditions publication bias was present with a $50 \%$ probability. That 258 means that $50 \%$ of the actors were willing to run additional analyses in order to obtain significant 259 results. These conditions seem closest to the behavioural benchmark of the empirical studies 260 presented.

261 If a non-significant result was obtained, actors operating under the second condition chose to 262 collect new data in order to obtain significant results that can be published. This second condition 263 therefore modelled publication bias under the file-drawer scenario, because the datasets not used 264 remained unpublished. An actor tried to run analyses on the basis of up to nine additional datasets 265 and only stopped earlier if a significant result with a positive sign was obtained. If none of the 10 266 datasets yielded a significant relationship with a positive sign, the estimate which was closest to 267 the significance threshold has been published. This rule served two purposes: firstly, it seemed 268 plausible that an actor who has tried that many analyses wants to get the results published in the 269 end to compensate for the invested effort and to avoid sunk costs (Thaler 1980). Secondly, from a 
270 technical point of view, this allowed to keep the number of observations in a meta-analysis $K$

271 constant across all simulation conditions.

272 In the third condition an actor did not try to achieve significant results by running the same

273 bivariate analysis on different samples, but rather tried to run different model specifications on

274 the same data by including control variables $\left(z_{j}\right)$ to achieve statistical significance of the 275 coefficient of interest $(\beta x)$. The third condition therefore modelled publication bias as a form of 276 -hacking, because the existing dataset was optimised to receive a significant $p$-value. The actor 277 was able to add three different control variables to the model. The control variables were defined 278 as collider variables that are both an effect of $x$ as well as $y$, which biases the effect of interest 279 (Cole et al. 2009; Greenland et al. 1999). The effect of $x$ and $y$ on $z_{j}$ was, however, only small $(\gamma=$ 280 0.5). The error term of the equation defining $z$ was normally distributed $N(0,10)$. With three 281 available control variables $z_{j}$ an actor had seven different combinations to improve the research 282 results in order to obtain a significant effect of $x$ on $y$.

283 In contrast to the second and third conditions, where $50 \%$ of the actors had the intention to 284 commit publication bias, in the fourth and fifth conditions all actors had the intention to engage in 285 publication bias practices, once again either through file-drawer (fourth condition) or p-hacking 286 behaviour (fifth condition). Part from the higher degree of intention to engage in publication bias 287 practices the settings remained the same. Although the two conditions where all actors had the 288 intent to engage in publication bias are far too pessimistic, they allow to evaluate the performance 289 of the tests in the most extreme publication bias environment. Tests that are not able to detect 290 publication bias even under such extreme conditions are of low utility to the research community.

291 The resulting design matrix had 100 different combinations resulting from 20 data setup 292 conditions multiplied by the five publication bias conditions. In order to obtain reliable estimates 
293 similarly to in an experiment (Carsey \& Harden 2013: 4f.), every single cell of the design matrix

294 had to be replicated multiple times. ${ }^{6}$

295 The aim of the simulation study at hand was to compare the performance of the four tests in

296 respect of: A) their capability to detect publication bias if present (true positive, statistical power),

297 as well as B) consistent false positive classification ( $\alpha$-error). Because the conditions with and

298 without publication bias are known in a simulation study, the power of the tests and the false

299 positive rate is computable (Mooney 1997: 77-79). In a first step, a dummy variable $(s)$ was

300 constructed, with the value 1 for a significant test result below the significance threshold $(5 \%$

301 significance level; $s=1$ if $p<0.05$ ). The statistical power, was than defined as the proportion

302 of significant results $s$ in respect to all runs with publication bias $\left(\sum_{i=1}^{r} s_{i} /\right.$ run if $P B>0$ ). The

303 false positive rate was computed equivalently but in conditions without publication bias (

$304 \quad \sum_{i=1}^{r} s_{i} /$ run if $P B=0 \quad$ ).

305 Results

6 In order to specify the number of replications that are necessary to achieve a sufficient statistical power of at least 80\% (Cohen 1988: 56) a power analysis was conducted for the statistical power, as well as the false positive rate estimates. For the false positive rate a small deviation of 1 percentage point from the set $5 \%$-false positive rate has to be correctly identified with at least an $80 \%$ chance. To achieve this goal, every condition without publication bias had to be supported with 3729 runs. As deviations in power are, though important, not as essential as the false positive rate (Cohen 1988: 56) a difference of 3 percentage points is set as acceptable. In order to identify a 3 percentage point deviation from the target power of $80 \%$ each of the 80 conditions with existing publication bias needed 1545 runs. In total, 198080 runs were necessary, resulting in nearly 109 million primary studies that in the case of publication bias contained up to 10 different regression models. 
306 Prevalence of publication bias

307 Because publication bias in the experimental setup was implemented as the intent to commit 308 publication bias, three variables are useful to address the actual publication bias and its impact on 309 the overall bias. Firstly, the share of actual studies per meta-analysis that suffer from publication 310 bias (if $p<0.05$ or negative result are obtained as a first result), secondly the share of studies that 311 achieve their goal of a significant positive result by publication bias, and thirdly the impact of 312 publication bias on the $p$-value of a fixed-effect meta-analysis (deflation factor of the $p$-value).

313 Because the heterogeneous effect condition of the simulation does not allow an absolute bias 314 measure the $p$-value deflation factor was used for all conditions.

315 In a first step the focus is on how the opportunity structures of the simulation conditions shape 316 the committed publication bias. In the first two columns of Table 3 the actual committed 317 publication bias is shown dependent on study characteristics like the mean number of 318 observations in the primary studies $(N)$ and the underlying effect $(\beta)$, including effect 319 heterogeneity. As expected around 50\% respectively $100 \%$ of the studies committed publication 320 bias in case of an underlying null-effect, because only $2.5 \%$ of the results had the right positive 321 sign and were significant just by chance. In case of an underlying effect the share of committed 322 publication bias decreased because an already significant finding made a publication bias 323 treatment unnecessary. For $\beta=0.5$ in the $50 \%$ publication bias condition only $35 \%$; for $\beta=1$, $32415 \%$ for $\beta=1.5$ only $9 \%$; and in the heterogeneous condition $22 \%$ of the studies employed 325 publication bias practices. The 100\% publication bias condition approximately doubled the 326 prevalence rates of the $50 \%$ condition as expected.

327 Besides the necessity of publication bias to achieve significant results also the success probability 328 in respect to committed publication bias depended on the conditions of the primary studies as 329 shown by the third column of Table 3 . For small studies $(N=100)$ with an underlying null-effect 
$330(\beta=0)$ the success-probability in respect to the committed publication bias was about $31.3 \%$.

331 Publication bias got more effective if larger studies $(N=500)$ provide the primary study with 332 more statistical power. The success probability of publication bias rose dramatically around 50 333 percentage points if a specific underlying empirical effect existed. Also in case of effect 334 heterogeneity the success probability increased about 35.8 percentage points. Slight differences 335 could be observed in the effectivity of the publication bias mechanism, as p-hacking was with 10 336 percentage points less effective than the file-drawer condition to achieve significant results.

337 As publication bias deflates $p$-values and therefore biases meta-analytical effect estimates the 338 impact of the actual observed publication bias (the share of committed and successful publication 339 bias) on the meta-analytical $p$-value is presented. The fourth column of Table 3 shows that with 340 an average proportion of publication bias committed (32.6\%) as well successfully implemented $341(18.8 \%)$ in a meta-analysis with 100 studies $(K=100)$ the $p$-value of the meta-analysis more than 342 quartered. This is further aggravated if the share of committed as well as successful publication 343 bias rose by 10 percentage points. The actual impact of successful publication bias deflated the $p$ 344 values by 7.7 percentage points and was more pronounced than the deflation caused by non345 successfully committed publication bias (deflation by 1.7 percentage points). The deflation also 346 was less severe if $p$-hacking procedures, as implemented in the simulation at hand, were used. 347 Nonetheless, the meta-analytical $p$-value in case of $p$-hacking is still less than half the size $348(42.2 \%)$ of the unbiased estimate.

349 False positive rate of publication bias tests

350 For the evaluated tests on publication bias consistent false positive rates are most important. In 351 the simulation none of the tests should exceed the prespecified 5\% error probability in any 352 condition. The false positive rate of the test was fixed in the simulation setting to 0.05 , so all false 
353 positive rates should be equal to, or even smaller than, 0.05 . Positive deviations from 0.05 point

354 to inflated false positive rates, which lead to more false conclusions than expected.

355 Table 4 shows the false positive rate in dependence of the number of studies included $(K=100$, 356 1000) and effect heterogeneity measured by $I^{2}$. In the constant condition of a meta-analysis with $357 K=100$ and no effect heterogeneity none of the tests had larger false positive rates than the 358 expected 0.05 . In particular, the TES, the $3 \%$ and $5 \%$ CTs were very conservative. A larger meta359 analytical sample increased the false positive rates for the TES and the CTs. The broadest $15 \%$ 360 CT missed the expected significance threshold of 5\%, with $7.8 \%$ clearly. The false positive rates 361 for PU in contrast were slightly lower. Increasing effect heterogeneity resulted in more 362 conservative false positive rates for PU, the $15 \% \mathrm{CT}$, and to a smaller extent also for the FAT, the 363 TES and the 10\% CT. The narrower 3\% and 5\% CTs were unaffected by effect heterogeneity.

364 The overall influence of the varied conditions on the false positive rate was small, as can be seen 365 by the small share of explained variance $\left(R^{2}<1.7 \%\right)$. Looking at the false positive rates by each 366 condition (Table A1 in the online appendix) only the 10\%- and 15\%-caliper showed increased 367 false positive rates because the underlying true effect rather than publication bias elicited an 368 overrepresentation of just significant values. Note however, that the $3 \%$ and $5 \%$ CTs showed now 369 increased false positive rates.

370 Statistical power of publication bias tests

371 The following regression model (Table 5) addresses the statistical power conditional on the type 372 of publication bias and its occurrence (committed as well as successful publication bias). Starting 373 from the baseline condition of a meta-analysis with $K=100$, a mean share of publication bias 374 committed (32.6\%), as well as successfully applied (18.8\%) via a file-drawer procedure and no 375 effect heterogeneity, the FAT had a superior power of $56.9 \%$, followed by the TES (51.5\%) and 376 the PU (48.3\%). The CTs performed worst and yielded only a power of $0.0 \%-38.6 \%$. 
377 The underperformance of the CTs is largely explained by the small number of studies in the meta-

378 analyses. With $K=100$ hardly any study falls within the small caliper around the significance

379 threshold. This limitation on just significant or non-significant effects also led to missing values,

380 because without observations in the caliper no CT could be performed. The underperformance of

381 the CT changed if 1000 studies were included, which improved the estimated power substantially,

382 by $30.7-57.3$ percentage points, while smaller calipers profited most. The FAT, the TES, and the

383 PU, profited moderately from an increased number of studies, by $24.4,23.8$ and 16.5 percentage

384 points, respectively. When focussing on the influence of heterogeneity in the meta-analyses the

385 PU and the TES showed a drastic drop in power, by 6.5 and 6.4 percentage points, if the

386 heterogeneity measured by $I^{2}$ rose by 10 percentage points. This decrease in power shows that

387 neither PU nor TES were able to cope with heterogeneity. In contrast, the FAT and the CTs

388 actually showed a slight increased statistical power. Varying the publication bias procedure from

389 file-drawer to p-hacking, which is less related to the standard error of the effect estimates,

390 increased the power of PU, TES, and the CTs. The CTs profited most, increasing the statistical

391 power by around 18 percentage points. The TES and PU showed a smaller increase of power, by

3927.5 and 4.8 percentage points. The FAT, in contrast lost about 11 percentage points of its power

393 under p-hacking compared to the file-drawer condition. Although the differences in power are

394 dependent especially on the operationalization of the p-hacking condition in the simulation, this

395 result points on a weakness of the FAT under non file-drawer conditions that are less related to

396 the standard error of the estimate but are still detectable in the distribution of $z$ - or $p$-values.

397 The structural difference between tests based on a continuous effect distribution (FAT, PU) and

398 tests that focus only on a dichotomous classification (TES, CTs) ${ }^{7}$ becomes evident looking at the

399 effect of the proportion of studies that underwent a publication bias treatment in the simulation

217 Significant or not (TES) over- or under-caliper (CTs). 
400 and the proportion of studies that had a successful outcome after publication bias. Increasing the 401 share of studies under publication bias lifted the power by 3.0 (FAT) and 5.1 (PU) percentage 402 points. A 10 percentage point increase in studies successfully applying publication bias increases 403 the power by 9.9 (FAT) and 10.3 percentage points (PU). The TES and the CTs, however, were 404 only able to detect successful publication bias. An increase only in studies committing 405 publication bias (whether successful or not) in contrast reduced the statistical power. Both tests 406 were therefore not able to detect all possible outcomes of publication bias. This is especially 407 problematic as non-successful publication bias may also inflate the overall estimated effect in 408 meta-analyses. All effects presented are statistically significant $(p<0.05)$.

409 In contrast to the influence of the varied conditions on the false positive rate, the influence on 410 statistical power was substantial, varying from $30.6 \%$ in the case of the FAT to $57.2 \%$ for the PU. 411 This finding underlines the fact that all publication bias tests have their strengths and weaknesses 412 in specific conditions.

\section{Discussion \& Conclusion}

414 In the simulation at hand, the performance of four different tests (PU, FAT, TES, CTs) were 415 evaluated in a Monte Carlo simulation. Different conditions were varied: the underlying true 416 effect size, including effect heterogeneity, the number of observations in the primary studies, the 417 number of studies in the meta-analyses, the degree of publication bias and its form as either file418 drawer or p-hacking.

419 Limitations

420 In order to compare the tests in a realistic setting that is nonetheless at least from the assumptions 421 of all four tests applicable, four central limitations have to be pointed out: 
422 Firstly the simulation and its according publication bias procedures rest on the assumptions that

423 all the correlation between the study's precision and its effect size is caused by publication bias.

424 In case that studies with larger effects are, for example after a pre-study power analysis (Lau et

425 al. 2006) conducted with a lower number of observation especially the FAT may yield increased

426 false positive rates (Schwarzer et al. 2002).

427 Secondly the number of observations included in the meta-analyses either set to $K=100$ or 1000

428 is large compared to the average meta-analysis (Elia et al. 2016). The results however showed

429 that even in such large meta-analyses and especially in the more realistic condition in which $50 \%$

430 of the actors are willing to commit publication bias, the tests hardly yielded an adequate

431 statistical power under most conditions. Increasing the number of included studies is therefore

432 important to assure an adequately powered test on publication bias.

433 Thirdly the analysis focused only on one specific form of p-hacking that could occur in both 434 small $(N=100)$ or large studies $(N=500)$. Especially for studies where $N$ is small, other 435 strategies like optional stopping may also be applied. Further research on publication bias should 436 therefore focus on the different impact of other $p$-hacking practices.

437 As a fourth limitation only one-sided publication bias against insignificant or negative results was 438 simulated. By assumption especially PU limits only on the negative or positive signed studies that 439 were supposed to be affected by publication bias. Also the FAT is not able to detect two-sided 440 publication bias because the funnel in this situation may still be perfectly symmetric. The 441 suggestions for applications if two-sided publication bias is suspected are therefore limited to the 442 TES and the CTs only.

443 Conclusion

444 The following five conclusions can be derived from the results: Firstly, for homogenous research 445 settings and with publication bias favouring only effects in one direction (one-sided publication 
446 bias) the FAT is recommended due to its most consistent false positive rate as well as its superior

447 statistical power. Secondly, if there are concerns whether there are any correlations between the 448 precision of the study and its effect size for other reasons than publication bias (see first 449 limitation) and if $p$-hacking is suspected, the TES should be preferred to the FAT under effect 450 homogeneity. As the 5\% CT offers more relaxed assumptions it is therefore the first alternative 451 for the FAT under effect heterogeneity if a large number of studies is included in the meta452 analysis.

453 Despite the analysis focussed only on one-sided publication bias, also two-sided publication bias, 454 favouring significant results with either sign may also be present. As PU and the FAT are not able 455 to identify one-sided publication bias only the TES and the CTs remain for two-sided publication 456 bias. Therefore, fourthly the TES is recommended under effect homogeneity because of its larger 457 statistical power compared to the CTs. Fifthly, in the case of heterogeneous effect sizes and a 458 sufficient number of observations in the meta-analysis the $5 \% \mathrm{CT}$ provides the best trade-off 459 between a conservative false positive rate and a decent statistical power.

460 The 5\% CT is therefore best used to identify publication bias in an effect heterogeneous 461 discipline-wide setting which relies per definition on completely different underlying effects but 462 offers enough studies to compensate for the low statistical power. Because the wider $10 \%$ and $46315 \%$ CTs yield inflated false positive rates, at least in some conditions, they are not recommended 464 to identify publication bias.

465 Identifying publication bias in substantial meta-analyses as well as focussing on publication as a 466 general problem within the scientific domain is necessary in order to establish and retain trust in 467 scientific results. Further research, however, should not only focus on the diagnosis of publication 468 bias just stating a problem that is well known (Morey 2013). Beyond the nonetheless important 469 diagnosis of the scientific "disease" a further examination of the risk factors, either on the side of 
470 the involved actors or with regard to the incentive structure within the discipline (see for example

471 Auspurg \& Hinz 2011) seem essential. This includes also the evaluation of possible interventions

472 (e.g. an open data policy). Research on publication bias is inevitable to maintain trust in scientific

473 results and avoid wasted research funds that also limit the efficiency of science at a whole.

474 Beside the diagnosis of publication bias and its risk factors, also estimators of the unbiased effect,

475 that are beyond the scope of this paper, like the effect estimates provided by PU and the PET (for

476 example the PET/PEESE procedure of Stanley \& Doucouliagos 2014) should be evaluated

477 comparatively. This is at most important for meta-analyses with a heterogeneous effect that try to

478 uncover the underlying true effect rather than test for publication bias alone.

\section{Acknowledgements}

480

481

482

483

484

485

486

487

488

489

490

491

492

493

494

495

496

497

I thank Katrin Auspurg and the two reviewers, Joseph Hilgard, Bob Reed and the responsible editor Robert Winkler for their valuable comments on this article.

\section{References}

Alinaghi N, and Reed WR. 2016. Meta-Analysis and Publication Bias: How Well Does the FATPET-PEESE Procedure Work? https://ideas.repec.org/p/cbt/econwp/16-26.html.

Auspurg K, and Hinz T. 2011. What Fuels Publication Bias? Theoretical and Empirical Analyses of Risk Factors Using the Caliper Test. Journal of Economics and Statistics 231:636-660.

Banks GC, Kepes S, and McDaniel MA. 2012. Publication Bias: A Call for Improved MetaAnalytic Practice in the Organizational Sciences. International Journal of Selection and Assessment 20:182-196. doi:10.1111/j.1468-2389.2012.00591.x.

Begg CB, and Mazumdar M. 1994. Operating Characteristics of a Bank Correlation Test for Publication Bias. Biometrics 50:1088-1101. doi:10.2307/2533446.

Bosco FA, Aguinis H, Singh K, Field JG, and Pierce CA. 2015. Correlational Effect Size Benchmarks. Journal of Applied Psychology 100:431-449. doi:10.1037/a0038047.

Brodeur A, Le M, Sangnier M, and Zylberberg Y. 2016. Star Wars: The Empirics Strike Back. American Economic Journal-Applied Economics 8:1-32. doi:10.1257/app.20150044.

Bürkner P-C, and Doebler P. 2014. Testing for Publication Bias in Diagnostic Meta-Analysis: A Simulation Study. Statistics in Medicine 33:3061-3077. doi:10.1002/sim.6177. 
498

499

500

501

502

503

504

505

506

507

508

509

510

511

512

513

514

515

516

517

518

519

520

521

522

523

524

525

526

527

528

529

530

531

532

533

534

535

536

537

538

539

540

541

542

543

544
Callaham ML, Wears RL, Weber EJ, Barton C, and Young G. 1998. Positive-Outcome Bias and Other Limitations in the Outcome of Research Abstracts Submitted to a Scientific Meeting. JAMA 280:254-257.

Carsey TM, and Harden JJ. 2013. Monte Carlo Simulation and Resampling Methods for Social Science: Sage Publications.

Chalmers 1. 1990. Underreporting Research is Scientific Misconduct. JAMA 263:1405-1408. doi:10.1001/jama.1990.03440100121018.

Cohen J. 1988. Statistical Power Analysis for the Behavioral Sciences. Hillsdale, N.J.: L. Erlbaum Associates.

Cohen J. 1992. A Power Primer. Psychological Bulletin 112:155-159.

Cohen J. 1994. The Earth Is Round ( $\mathrm{p}<.05)$. American Psychologist 49:997-1003. doi:10.1037/0003-066x.49.12.997.

Cole SR, Platt RW, Schisterman EF, Chu H, Westreich D, Richardson D, and Poole C. 2009. Illustrating Bias Due to Conditioning on a Collider. International Journal of Epidemiology 39:417-420. doi:10.1093/ije/dyp334.

Coursol A, and Wagner EE. 1986. Effect of Positive Findings on Submission and Acceptance: A Note of Meta-Analysis Bias. Professional Psychology: Research and Practice 17. doi:10.1037/0735-7028.17.2.136.

Descartes R. 2006. A Discourse on the Method of Correctly Conducting One's Reason and Seeking Truth in the Sciences. Oxford ; New York: Oxford University Press.

Dickersin K. 1990. The Existence of Publication Bias and Risk Factors for Its Occurrence. JAMA 263:1385-1389. doi:10.1001/jama.1990.03440100097014.

Dickersin K, and Min Y-I. 1993. Publication Bias - the Problem that Won't Go Away. Annals of the New York Academy of Sciences 703:135-148. doi:10.1111/j.17496632.1993.tb26343.x.

Doucouliagos H, and Stanley TD. 2009. Publication Selection Bias in Minimum-Wage Research? A Meta-Regression Analysis. British Journal of Industrial Relations 47:406-428. doi:10.1111/j.1467-8543.2009.00723.x.

Duval S, and Tweedie R. 2000. Trim and Fill: A Simple Funnel-Plot-Based Method of Testing and Adjusting for Publication Bias in Meta-Analysis. Biometrics 56:455-463. doi:10.1111/j.0006-341X.2000.00455.x.

Easterbrook PJ, Berlin JA, Gopalan R, and Matthews DR. 1991. Publication Bias in Clinical Research. Lancet 337:867-872.

Egger M, Smith GD, Schneider M, and Minder C. 1997. Bias in Meta-Analysis Detected by a Simple, Graphical Test. British Medical Journal 315:629-634.

Elia N, von Elm E, Chatagner A, Pöpping DM, and Tramèr MR. 2016. How Do Authors of Systematic Reviews Deal with Research Malpractice and Misconduct in Original Studies? a Cross-Sectional Analysis of Systematic Reviews and Survey of Their Authors. BMJ open 6. doi:10.1136/bmjopen-2015-010442.

Epstein WM. 1990. Confirmational Response Bias among Social-Work Journals. Science Technology \& Human Values 15:9-38. doi:10.1177/016224399001500102.

Epstein WM. 2004. Confirmational Response Bias and the Quality of the Editorial Processes among American Social Work Journals. Research on Social Work Practice 14:450-458. doi:10.1177/1049731504265838.

Ferguson CJ, and Brannick MT. 2012. Publication Bias in Psychological Science: Prevalence, Methods for Identifying and Controlling, and Implications for the Use of Meta-Analyses. Psychological Methods 17:120-128. doi:10.1037/a0024445. 
545 Ferguson CJ, and Heene M. 2012. A Vast Graveyard of Undead Theories: Publication Bias and

546

547

548

549

550

551

552

553

554

555

556

557

558

559

560

561

562

563

564

565

566

567

568

569

570

571

572

573

574

575

576

577

578

579

580

581

582

583

584

585

586

587

588

589

590

591

592

593
Psychological Science's Aversion to the Null. Perspectives on Psychological Science

7:555-561. doi:10.1177/1745691612459059.

Francis G. 2012a. Evidence That Publication Bias Contaminated Studies Relating Social Class and Unethical Behavior. Proceedings of the National Academy of Sciences of the United States of America 109:E1587-E1587. doi:10.1073/pnas.1203591109.

Francis G. 2012b. The Psychology of Replication and Replication in Psychology. Perspectives on Psychological Science 7:585-594. doi:10.1177/1745691612459520.

Francis G. 2012c. Publication Bias and the Failure of Replication in Experimental Psychology. Psychonomic Bulletin \& Review 19:975-991. doi:10.3758/s13423-012-0322-y.

Francis G. 2012d. The Same Old New Look: Publication Bias in a Study of Wishful Seeing. $i$ Perception 3:176-178. doi:10.1068/i0519ic.

Francis G. 2012e. Too Good to Be True: Publication Bias in Two Prominent Studies from Experimental Psychology. Psychonomic Bulletin \& Review 19:151-156. doi:10.3758/s13423-012-0227-9.

Francis G. 2013. Publication Bias in 'Red, Rank, and Romance in Women Viewing Men,' by Elliot et al. (2010). Journal of Experimental Psychology: General 142:292-296. doi:10.1037/a0027923.

Franco A, Malhotra N, and Simonovits G. 2014. Publication Bias in the Social Sciences: Unlocking the File Drawer. Science. doi:10.1126/science.1255484.

Gelman A. 2013. Too Good to Be True. Available at http://www.slate.com/articles/health and science/science/2013/07/statistics and psychol ogy multiple comparisons give spurious results.html (accessed 6.1.2017).

Gerber AS, and Malhotra N. 2008a. Do Statistical Reporting Standards Affect What Is Published? Publication Bias in Two Leading Political Science Journals. Quarterly Journal of Political Science 3:313-326. doi:10.1561/100.00008024.

Gerber AS, and Malhotra N. 2008b. Publication Bias in Empirical Sociological Research. Sociological Methods \& Research 37:3-30. doi:10.1177/0049124108318973.

Godlee F. 2012. Research Misconduct is Widespread and Harms Patients. BMJ 344:e:14. doi:10.1136/bmj.e14.

Greenland S, Pearl J, and Robins JM. 1999. Causal Diagrams for Epidemiologic Research. Epidemiology 10:37-48. doi:10.1097/00001648-199901000-00008.

Hayashino Y, Noguchi Y, and Fukui T. 2005. Systematic Evaluation and Comparison of Statistical Tests for Publication Bias. Journal of Epidemiology 15:235-243. doi:10.2188/jea.15.235.

Higgins JPT, and Green S. 2008. Cochrane Handbook for Systematic Reviews of Interventions: Wiley Online Library.

Higgins JPT, and Thompson SG. 2002. Quantifying Heterogeneity in a Meta-Analysis. Statistics in Medicine 21:1539-1558. doi:10.1002/sim.1186.

Ioannidis JP. 1998. Effect of the Statistical Significance of Results on the Time to Completion and Publication of Randomized Efficacy Trials. JAMA 279:281-286. doi:10.1001/jama.279.4.281.

Ioannidis JPA. 2013. Clarifications on the Application and Interpretation of the Test for Excess Significance and Its Extensions. Journal of Mathematical Psychology 57:184-187. doi:10.1016/j.jmp.2013.03.002.

Ioannidis JPA, Stanley TD, and Doucouliagos H. 2017. The Power of Bias in Economics Research. The Economic Journal 127:F236-F265. doi:10.1111/ecoj.12461.

Ioannidis JPA, and Trikalinos TA. 2007. An Exploratory Test for an Excess of Significant Findings. Clinical Trials 4:245-253. doi:10.1177/1740774507079441. 
594

595

596

597

598

599

600

601

602

603

604

605

606

607

608

609

610

611

612

613

614

615

616

617

618

619

620

621

622

623

624

625

626

627

628

629

630

631

632

633

634

635

636

637

638

639

640

641

Jefferson T, Jones MA, Doshi P, Del Mar CB, Heneghan CJ, Hama R, and Thompson MJ. 2012. Neuraminidase Inhibitors for Preventing and Treating Influenza in Healthy Adults. Cochrane database of systematic reviews. doi:10.1002/14651858.CD008965.pub3.

John LK, Loewenstein G, and Prelec D. 2012. Measuring the Prevalence of Questionable Research Practices With Incentives for Truth Telling. Psychological Science 23:524-532. doi:10.1177/0956797611430953.

Kerr NL. 1998. HARKing: Hypothesizing After the Results are Known. Personality and Social Psychology Review 2:196-217. doi:10.1207/s15327957pspr0203_4.

Kicinski M. 2014. How Does Under-Reporting of Negative and Inconclusive Results Affect the False-Positive Rate in Meta-Analysis? A Simulation Study. BMJ open 4:1-8. doi:10.1136/bmjopen-2014-004831.

Kreuter F, Presser S, and Tourangeau R. 2008. Social Desirability Bias in CATI, IVR, and Web Surveys: The Effects of Mode and Question Sensitivity. Public Opinion Quarterly 72:847-865. doi:10.1093/poq/nfn063.

Labovitz S. 1972. Statistical Usage In Sociology: Sacred Cows and Ritual. Sociological Methods \& Research 1:13-37. doi:10.1177/004912417200100102.

Lau J, Ioannidis JPA, Terrin N, Schmid CH, and Olkin I. 2006. The Case of the Misleading Funnel Plot. BMJ 333:597-600. doi:10.1136/bmj.333.7568.597.

Macaskill P, Walter SD, and Irwig L. 2001. A Comparison of Methods to Detect Publication Bias in Meta-Analysis. Statistics in Medicine 20:641-654. doi:10.1002/sim.698.

Mahoney MJ. 1977. Publication Prejudices: An Experimental Study of Confirmatory Bias in the Peer Review System. Cognitive Therapy and Research 1:161-175. doi:10.1007/BF01173636.

Mallett S, and Clarke M. 2002. The Typical Cochrane Review. How Many Trials? How Many Participants? International Journal of Technology Assessment in Health Care 18:820-823.

Merton RK. 1957. Priorities in Scientific Discovery: A Chapter in the Sociology of Science. American sociological review 22:635-659. doi:10.2307/2089193.

Mooney CZ. 1997. Monte Carlo Simulation. Thousand Oakes: Sage Publications.

Moreno SG, Sutton AJ, Ades AE, Stanley TD, Abrams KR, Peters JL, and Cooper NJ. 2009. Assessment of Regression-based Methods to Adjust for Publication Bias through a Comprehensive Simulation Study. Bmc Medical Research Methodology 9. doi:10.1186/1471-2288-9-2.

Morey RD. 2013. The Consistency Test Does Not - and Cannot - Deliver What Is Advertised: a Comment on Francis (2013). Journal of Mathematical Psychology 57:180-183. doi:10.1016/j.jmp.2013.03.004.

Nature. 2017. Getting Published in Nature: The Editorial Process. Available at http://www.nature.com/nature/authors/get published/ (accessed 05.01.2017).

Nuzzo R. 2014. Scientific Method: Statistical Errors. Nature 506:150-152. doi:10.1038/506150a.

Paldam M. 2013. Regression Costs Fall, Mining Ratios Rise, Publication Bias Looms, and Techniques Get Fancier: Reflections on Some Trends in Empirical Macroeconomics. Econ Journal Watch 10:136-156.

Paldam M. 2015. Simulating an Empirical Paper by the Rational Economist. Empirical Economics:1-25. doi:10.1007/s00181-015-0971-6.

Popper KR. 1968. Conjectures and Refutations: The Growth of Scientific Knowledge. New York: Harper \& Row.

Renkewitz F, and Keiner M. 2016. How to Detect Publication Biases from Published Data? A Monte Carlo Simulation of Different Methods. 50 Kongress der Deutschen Gesellschaft für Psychologie. Leipzig. 
642

643

644

645

646

647

648

649

650

651

652

653

654

655

656

657

658

659

660

661

662

663

664

665

666

667

668

669

670

671

672

673

674

675

676

677

678

679

680

681

682

683

684

685

686

687

688
Rosenthal R. 1979. The File Drawer Problem and Tolerance for Null Results. Psychological Bulletin 86:638-641. doi:10.1037/0033-2909.86.3.638.

Schimmack U. 2012. The Ironic Effect of Significant Results on the Credibility of MultipleStudy Articles. Psychological Methods 17:551-566. doi:10.1037/a0029487.

Schwarzer G, Antes G, and Schumacher M. 2002. Nflation of Type I Error Rate in Two Statistical Tests for the Detection of Publication Bias in Meta-Analyses with Binary Outcomes. Statistics in Medicine 21:2465-2477. doi:10.1002/sim.1224.

Science. 2017. The Science Contributors FAQ. Available at http://www.sciencemag.org/site/feature/contribinfo/faq/\#pct faq (accessed 05.01.2017).

Simmons JP, Nelson LD, and Simonsohn U. 2011. False-Positive Psychology: Undisclosed Flexibility in Data Collection and Analysis Allows Presenting Anything as Significant. Psychological Science 22:1359-1366. doi:10.1177/0956797611417632.

Simonsohn U. 2013. It Really Just Does Not Follow, Comments on Francis (2013). Journal of Mathematical Psychology 57:174-176. doi:10.1016/j.jmp.2013.03.006.

Simonsohn U, Nelson LD, and Simmons JP. 2014a. P-Curve and Effect Size: Correcting for Publication Bias Using Only Significant Results. Perspectives on Psychological Science 9:666-681. doi:10.1177/1745691614553988.

Simonsohn U, Nelson LD, and Simmons JP. 2014b. P-curve: A Key to the File Drawer. Journal of Experimental Psychology-General 143:534-547. doi:10.1037/a0033242.

Simonsohn U, Simmons JP, and Nelson LD. 2015. Better P-Curves: Making P-Curve Analysis More Robust To Errors, Fraud, and Ambitious P-Hacking, A Reply To Ulrich and Miller (2015). Journal of Experimental Psychology-General 144:1146-1152. doi:10.1037/xge0000104.

Stanley TD, and Doucouliagos H. 2014. Meta-Regression Approximations to Reduce Publication Selection Bias. Research Synthesis Methods 5:60-78. doi:10.1002/jrsm.1095.

Sterne JAC, Gavaghan D, and Egger M. 2000. Publication and Related Bias in Meta-Analysis: Power of Statistical Tests and Prevalence in the Literature. Journal of Clinical Epidemiology 53:1119-1129. doi:10.1016/S0895-4356(00)00242-0.

Sterne JAC, Sutton AJ, Ioannidis JPA, Terrin N, Jones DR, Lau J, Carpenter J, Rücker G, Harbord RM, Schmid CH, Tetzlaff J, Deeks JJ, Peters J, Macaskill P, Schwarzer G, Duval S, Altman DG, Moher D, and Higgins JPT. 2011. Recommendations for examining and interpreting funnel plot asymmetry in meta-analyses of randomised controlled trials. $B M J$ 343. doi:10.1136/bmj.d4002.

Terrin N, Schmid CH, Lau J, and Olkin I. 2003. Adjusting for Publication Bias in the Presence of Heterogeneity. Statistics in Medicine 22:2113-2126. doi:10.1002/sim.1461.

Thaler R. 1980. Toward a Positive Theory of Consumer Choice. Journal of Economic Behavior \& Organization 1:39-60. doi:10.1016/0167-2681(80)90051-7.

Ulrich R, and Miller J. 2017. Some Properties of p-Curves, With an Application to Gradual Publication Bias. Psychological Methods. doi:10.1037/met0000125.

van Aert RCM, Wicherts JM, and van Assen MALM. 2016. Conducting Meta-Analyses Based on p Values. Perspectives on Psychological Science 11:713-729. doi:10.1177/1745691616650874.

van Assen MALM, van Aert RCM, and Wicherts JM. 2015. Meta-Analysis Using Effect Size Distributions of only Statistically Significant Studies. Psychological Methods 20:293-309. doi:10.1037/met0000025 10.1037/met0000025.supp (Supplemental).

Wasserstein RL, and Lazar NA. 2016. The ASA's Statement on p-Values: Context, Process, and Purpose. American Statistician 70:129-131. doi:10.1080/00031305.2016.1154108. 
689 Yoder S, and Bramlett BH. 2011. What Happens at the Journal Office Stays at the Journal Office:

690 Assessing Journal Transparency and Record-Keeping Practices. Ps-Political Science \& Politics 44:363-373. doi:10.1017/S1049096511000217. 


\section{Table $\mathbf{1}$ (on next page)}

Publication bias tests in comparison

Table 1 compares the four evaluated publication bias tests in respect to four criteria, the measurement level, the sample used by the test, its underlying assumptions and its limitations. 


\begin{tabular}{|c|c|c|c|c|}
\hline Test & $\begin{array}{l}\text { Measurement } \\
\text { level }\end{array}$ & Sample & Assumption & Limitation \\
\hline FAT & $\begin{array}{l}\text { Continuous } \\
{[-\infty, \infty]}\end{array}$ & All & $\operatorname{Cov}(\mathrm{es}, \mathrm{se})=0$ & $\begin{array}{l}\text { Only one-sided publication bias } \\
\text { (PB) detectable }\end{array}$ \\
\hline PU & Continuous $[0,1]$ & $\begin{array}{l}p<0.05 \\
\text { effects of } \\
\text { same sign }\end{array}$ & $\begin{array}{l}\text { Uniform or right } \\
\text { skewed } \\
\text { Skewness }>=0\end{array}$ & $\begin{array}{l}\text { Only one-sided PB detectable } \\
\text { Only on prespecified levels } \\
\text { Effect homogeneity (fixed- } \\
\text { effect meta-analysis) }\end{array}$ \\
\hline TES & $\begin{array}{l}\text { Dichotomous } \\
{[0,1]}\end{array}$ & All & $\mathrm{E}=\mathrm{O}$ & $\begin{array}{l}\text { Only on prespecified levels } \\
\text { Effect homogeneity (fixed- } \\
\text { effect meta-analysis) }\end{array}$ \\
\hline $\mathrm{CT}$ & $\begin{array}{l}\text { Dichotomous } \\
{[0,1]}\end{array}$ & $\begin{array}{l}\text { Threshold } \\
\pm \text { caliper } \\
\text { width }\end{array}$ & $\mathrm{P}(\mathrm{UC})=\mathrm{P}(\mathrm{OC})$ & Only on prespecified levels \\
\hline
\end{tabular}




\section{Table 2 (on next page)}

Data generating process (DGP) of Monte Carlo simulation

Table 2 describes the 100 conditions of the Monte Carlo simulation. Two different aspects were varied: the underlying data and the publication bias behaviour of the actors. For the underlying data the true effect size, the number of observations $(N)$ and the number of studies included in the meta-analysis $(K)$ were varied. The behavioural component altered the proportions of authors who are willing to commit publication bias and its actual form as either p-hacking or file-drawer. 
Conditions

Data setup:

1. True effects $\beta$ :

2. Number of observations $N$ :

3. Number of studies $K$

Behavioural setup:

4. Publication bias (PB)

4.1.File-drawer

4.2.p-hacking
Values

$\beta=0 ; 0.5 ; 1 ; 1.5 ;$ Het

$\varepsilon=\mathrm{NV}(0,10)$

$\sigma_{x}=2$

$\mu_{N}=100 ; 500$

$K=100 ; 1000$

$\left|\mathrm{N}\left(\mu_{N}\right)\right| N>30$

$1+2 * 2=5$

(max. 9 additional samples)

$\mathrm{y}=\beta \mathrm{x}+\gamma_{\mathrm{j}} \mathrm{z}_{\mathrm{j}}+\varepsilon$

$\mathrm{z}=0.5 \mathrm{x}+0.5 \mathrm{y}+\varepsilon$

(max. $3 z$ 's $=7$

combinations)

\section{$N$ (conditions)}

5

Run new analyses with same dataset 


\section{Table 3 (on next page)}

Risk factors for publication and its impact on bias in the simulated data (OLS regression)

The first two columns in Table 3 show that actual committed publication bias behaviour depended largely on the opportunity structure of the underlying data. Despite the defined $50 \%$ or $100 \%$ willingness of the authors to commit publication bias, only those actors who face insignificant effects (caused by small effects and sample sizes) engaged in publication bias practices. The success of publication bias in terms of significant results is shown in column three, dependent on the opportunity structure and form of publication bias. Conditions under $p$-hacking were slightly less effective in obtaining significant results than conditions under file-drawer publication bias. Column four shows the deflating impact of publication bias on meta-analytic $p$-values. For an average publication bias this $p$-values halved or even quartered. 


\begin{tabular}{|c|c|c|c|c|}
\hline & $\begin{array}{c}\text { Publication bias } \\
\text { committed ( } 50 \% \\
\text { intention) }\end{array}$ & $\begin{array}{l}\text { Publication bias } \\
\text { committed (100\% } \\
\text { intention) }\end{array}$ & $\begin{array}{l}\text { Publication bias } \\
\text { successful (in } \\
\text { relation to } \\
\text { committed) } \\
\end{array}$ & $\begin{array}{l}\text { Deflation of } p \text { - } \\
\text { value }\end{array}$ \\
\hline$N=500$ & $-0.105 * * *$ & $-0.211 * * *$ & $0.179 * * *$ & \\
\hline$($ ref. $N=100)$ & $(0.000)$ & $(0.001)$ & $(0.001)$ & \\
\hline$\beta=0.5$ & $-0.196^{* * *}$ & $-0.391 * * *$ & $0.471 * * *$ & \\
\hline (ref. $\beta=0$ ) & $(0.001)$ & $(0.001)$ & $(0.001)$ & \\
\hline \multirow[t]{2}{*}{$\beta=1$} & $-0.389 * * *$ & $-0.777 * * *$ & $0.513 * * *$ & \\
\hline & $(0.001)$ & $(0.001)$ & $(0.001)$ & \\
\hline \multirow[t]{2}{*}{$\beta=1.5$} & $-0.451 * * *$ & $-0.899 * * *$ & $0.503 * * *$ & \\
\hline & $(0.001)$ & $(0.001)$ & $(0.002)$ & \\
\hline \multirow[t]{2}{*}{$\beta=$ heterogeneous } & $-0.319 * * *$ & $-0.636^{* * *}$ & $0.358 * * *$ & \\
\hline & $(0.001)$ & $(0.001)$ & $(0.001)$ & \\
\hline p-hacking & & & $-0.100 * * *$ & $0.197 * * *$ \\
\hline (ref. file-drawer) & & & $(0.001)$ & $(0.002)$ \\
\hline Committed PB [+10ppts] & & & & $-0.018 * * *$ \\
\hline (ref. mean $=32.5 \%)$ & & & & $(0.001)$ \\
\hline Successful PB [+10ppts] & & & & $-0.077 * * *$ \\
\hline (ref. mean $=18.8 \%)$ & & & & $(0.001)$ \\
\hline \multirow[t]{2}{*}{ Constant } & $0.541 * * *$ & $1.080 * * *$ & $0.313 * * *$ & $0.225 * * *$ \\
\hline & $(0.000)$ & $(0.001)$ & $(0.001)$ & $(0.002)$ \\
\hline Observations & 61,760 & 61,760 & 115,843 & 123,520 \\
\hline $\mathrm{R}^{2}$ & 0.939 & 0.958 & 0.648 & 0.168 \\
\hline
\end{tabular}

Standard errors in parentheses; ${ }^{* * *} \mathrm{p}<0.001,{ }^{* *} \mathrm{p}<0.01,{ }^{*} \mathrm{p}<0.05$ 


\section{Table 4(on next page)}

Conditional false positive rates of the publication bias tests (OLS regression)

Table 4 displays the false positive rates of the publication bias tests conditional on the number of studies included in the meta-analysis $(K)$ as well as the between study heterogeneity $\left(\mathrm{I}^{2}\right)$. The FAT had the most consistent false positive rate. The $15 \%$ CT missed the $5 \%$-level clearly while the $10 \%$ CT showed a large variability and gets close to it. The $10 \%$ and $15 \% \mathrm{CT}$ are therefore problematic because they may suffer from inflated false positive rates. 


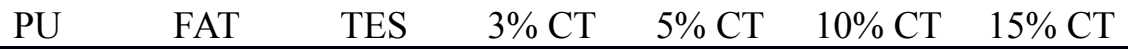

\begin{tabular}{llllllll}
$K=1000$ & $-0.005^{* * *}$ & 0.000 & $0.006^{* * *}$ & $0.026^{* * *}$ & $0.023^{* * *}$ & $0.026^{* * *}$ & $0.050^{* * *}$ \\
(ref. $K=100)$ & $(0.001)$ & $(0.002)$ & $(0.001)$ & $(0.001)$ & $(0.001)$ & $(0.001)$ & $(0.002)$ \\
$\mathrm{I}^{2}[+10$ percentage points] $]$ & $-0.003^{* * *}$ & $-0.001^{* * *}$ & $-0.001^{* * *}$ & 0.000 & 0.000 & $-0.001^{* * *}$ & $-0.003^{* * *}$ \\
& $(0.000)$ & $(0.000)$ & $(0.000)$ & $(0.000)$ & $(0.000)$ & $(0.000)$ & $(0.000)$ \\
Constant $^{\mathrm{a}}$ & $0.023^{* * *}$ & 0.049 & $0.010^{* * *}$ & $0.003^{* *}$ & $0.008^{* * *}$ & $0.019^{* * * *}$ & $0.028^{* * *}$ \\
& $(0.001)$ & $(0.001)$ & $(0.001)$ & $(0.001)$ & $(0.001)$ & $(0.001)$ & $(0.001)$ \\
& & & & & & & \\
Observations & 73,960 & 74,560 & 74,560 & 62,644 & 66,546 & 69,718 & 70,936 \\
$\mathrm{R}^{2}$ & 0.005 & 0.000 & 0.002 & 0.010 & 0.007 & 0.006 & 0.017 \\
\hline
\end{tabular}

${ }^{\mathrm{a}}$ Test H0: constant $=0.05$; Standard errors in parentheses; ${ }^{* *} \mathrm{p}<0.001,{ }^{* *} \mathrm{p}<0.01,{ }^{*} \mathrm{p}<0.05$ 


\section{Table 5 (on next page)}

Conditional statistical power of the publication bias tests (OLS regression)

Table 5 shows the statistical power of the publication bias tests conditional on the number of studies included in the meta-analysis $(K)$ and the between study heterogeneity $\left(I^{2}\right)$. In contrast to Table 4, also the share of committed as well as successful publication bias and its form as either file-drawer or p-hacking was controlled. Overall the FAT had the largest power but was not able to detect $p$-hacking as good as the TES. The CTs were underpowered if a low number of studies was included in a meta-analysis but performed well in studies with large Ks. Both, PU and the TES, were not able to detect publication bias under effect heterogeneity. 


\begin{tabular}{llllllll} 
& \multicolumn{1}{c}{$\mathrm{PU}$} & $\mathrm{FAT}$ & \multicolumn{1}{c}{$\mathrm{TES}$} & $3 \% \mathrm{CT}$ & $5 \% \mathrm{CT}$ & $10 \% \mathrm{CT}$ & $15 \% \mathrm{CT}$ \\
\hline & & & & & & & \\
$K=1000$ & $0.165^{* * *}$ & $0.244^{* * *}$ & $0.238^{* * *}$ & $0.573^{* * *}$ & $0.513^{* * *}$ & $0.382^{* * *}$ & $0.307^{* * *}$ \\
(ref. $K=100)$ & $(0.002)$ & $(0.002)$ & $(0.002)$ & $(0.002)$ & $(0.002)$ & $(0.002)$ & $(0.002)$ \\
$\mathrm{I}^{2}[+10$ percentage points] & $-0.065^{* * *}$ & $0.001^{*}$ & $-0.064^{* * *}$ & $0.006^{* * *}$ & $0.005^{* * *}$ & $0.008^{* * *}$ & $0.010^{* * *}$ \\
& $(0.000)$ & $(0.000)$ & $(0.000)$ & $(0.000)$ & $(0.000)$ & $(0.000)$ & $(0.000)$ \\
& $0.048^{* * *}$ & $-0.110^{* * *}$ & $0.075^{* * *}$ & $0.179^{* * *}$ & $0.187^{* * *}$ & $0.186^{* * *}$ & $0.177^{* * *}$ \\
(ref. file-drawer) & $(0.002)$ & $(0.002)$ & $(0.002)$ & $(0.002)$ & $(0.002)$ & $(0.002)$ & $(0.002)$ \\
Comitted PB [+10ppts] & $0.051^{* * *}$ & $0.030^{* * *}$ & $-0.065^{* * *}$ & $-0.035^{* * *}$ & $-0.053^{* * *}$ & $-0.073^{* * *}$ & $-0.084^{* * *}$ \\
(ref. mean = 32.6\%) & $(0.000)$ & $(0.001)$ & $(0.001)$ & $(0.001)$ & $(0.001)$ & $(0.001)$ & $(0.001)$ \\
Successful PB [+10ppts] & $0.103^{* * *}$ & $0.099^{* * *}$ & $0.221^{* * *}$ & $0.162^{* * *}$ & $0.193^{* * *}$ & $0.224^{* * *}$ & $0.234^{* * *}$ \\
(ref. mean $=18.8 \%)$ & $(0.001)$ & $(0.001)$ & $(0.001)$ & $(0.001)$ & $(0.001)$ & $(0.001)$ & $(0.001)$ \\
Constant ${ }^{2}$ & $0.483^{* * *}$ & $0.569^{* * *}$ & $0.515^{* * *}$ & -0.002 & $0.125^{* * *}$ & $0.300^{* * *}$ & $0.386^{* * *}$ \\
& $(0.002)$ & $(0.002)$ & $(0.002)$ & $(0.002)$ & $(0.002)$ & $(0.002)$ & $(0.002)$ \\
& & & & & & & \\
Observations & 123,520 & 123,520 & 123,520 & 107,736 & 111,315 & 115,243 & 117,207 \\
$\mathrm{R}^{2}$ & 0.572 & 0.306 & 0.473 & 0.497 & 0.483 & 0.457 & 0.446 \\
\hline
\end{tabular}

${ }^{a}$ Test H0: constant $=0.8$; Standard errors in parentheses; ${ }^{* * *} \mathrm{p}<0.001,{ }^{*} * \mathrm{p}<0.01,{ }^{*} \mathrm{p}<0.05$ 